\title{
EMBEDDING ARTIFICIAL INTELLIGENCE IN HOSPITALITY \& TOURISM
}

\author{
${ }^{1}$ Dr.Sameer Koranne, ${ }^{2}$ Sukhmani Sandhu \\ ${ }^{1}$ Associate Dean-Galgotias University, Greater Noida, U.P. \\ ${ }^{2}$ Assistant Professor, Chandigarh Business School of Administration, CGC, Landran, Mohali \\ spkoranne@gmail.com ORCID: 0000-0002-6917-2391
}

\begin{abstract}
:
Competitive environment and being global trade; the tourism and hospitality industry adopted digital technology a long back and many functions have been digitalized. The travelling guest leaves digital foot prints across various components of tourism and thus a huge amount of data is generated. The customer satisfaction has been an integral part of this industry and providing appropriate service solutions is vital for excelling. Artificial Intelligence is utilized for exploring and analyzing guest data and compliments decision making. The effective existence of AI across various tourism and hospitality functions is investigated through empirical study and validated through descriptive analysis.
\end{abstract}

KEYWORDS: Artificial intelligence, Tourism \& Hospitality operations, Decision making, Service solutions

Article Received: 10 August 2020, Revised: 25 October 2020, Accepted: 18 November 2020

\section{INTRODUCTION}

Since the globalization along with advent of internet and digital world, the travelers leaves its digital footprints at cab service, airports, airport lounges, airport shopping center, airlines, payment gateways, hotels, tour agencies, local bus services, rail services, guide services, monuments and museums, small eateries, shopping plazas, money exchange and every touch point of tourism. Even though the guests leave their digital footprints throughout their travel, it is found that the conversion of these footprints into actionable insights is very low. Due to the enormous data stored at different locations and in variety of forms, AI is most needed technology for converting the data into suitable insights.

Tourism and hospitality is a global industry and has always witnessed the changes in technology a long back. This industry is working seamlessly with its counterparts all across the world. Technology has proved to an important factor in expansion and modernization of this industry. Tourism and hospitality industry was forerunner in reaping the benefits of digital technology. Artificial intelligence (AI) and Machine Learning (ML) is adopted a long back in day to day operations. According to IDC hospitality prediction (2018), AI is would have been adopted by more than $70 \%$ of travel and hospitality companies with an additional increase of customer base by $30 \%$. According to SITA Air Transport Insights report (2017), 52\% of Airlines and $45 \%$ of airport are likely to deploy AIML in coming couple of years.

Artificial intelligence has reshaped the operations of in manifolds and it has helped the industry grow to the global standards of service quality. Artificial intelligence opens up the opportunity for enhanced marketing, highest service quality, improved customer experience and higher rate of retention. It has provided an exceptional ability to have insights in the patterns of customer requirements and it can handle abundant data for providing the finest hospitality solutions. It also empowers the enterprise to analyze the large amount of data which is freely available at different sources. It becomes imperative to know and analyze the advantages of AI in overall management of travel \& hospitality sector. This study examines important areas where AI has transformed way of operations and positive effects on management of hospitality business. However, a explicit study of AI across various functions of hotel and travel operations is yet to be ascertained. The specific objective of the study is examining the implantation of AI in tourism and hotel operations and its effectiveness in various functions.

\section{Literature review}

Minsky Marvin, (1961) has confirmed that the computer programs are based on five elements that include Searching, Recognizing the pattern, Learning, Planning the action and Induction. Though it does not do it on its own, but can be made to do 
certain things by programming it as per the need. Today, the technology of Artificial Intelligence is being used more often in different sectors of businesses and has gradually become integral part of our lives. As per Frankenfield, (2020), the term Artificial Intelligence literally means "the simulation of human intelligence in machines that are programmed to think like humans and mimic their actions". Author John Paul Mueller (2018) said that AI must be made of some core properties; 'Acting humanly, thinking humanly, thinking rationally, and acting rationally'. As per Kaplan (2016), the intelligence part is a capability to make suitable generalizations in a time bound fashion based upon given data. This definition is most suitable for hospitality industry as guest provides limited and restricted information; it may in terms of feedback or comment. This may also include some qualitative data generated through articulate surveys. Wang Swien (2020) found that hotel chains and large hospitality companies are creating their own chatboats, robots and self help kiosks.Marriot has incented its own robot in 2015 whereas the Aloft hotel company has its own chatboat in year 2014. A Japanese firm named Henna operated with robots without any human intervention. IT majors like IBM and Toshiba are spearheading the usage on technology in hospitality industry.

Hensens (2015) mentioned that the hotel ratings system is now being dictated by guest feedback in contrast to earlier system of government regulatory bodies. The best and most reliable hotel ratings are found on tripadvisor.com, which are derived from the actual feedback of users. Thanks to the use of internet, travel websites and applications used for travel booking, huge amount of data is generated all over the world and AI is used to systematic analysis of the data for appropriate inferences.

Sameer K (2020) has mentioned that hospitality industry has highest regard for service quality. It has witnessed changes manifold and to service quality is only instrument for creating the differentiator in the competition. Service quality is an integral part of overall service operations and has been catering ever demanding global customer. The customer expectation of services and their perception of service delivery is recorded for deriving the numbers of service quality.

AI is quintessentially based on data mining. The complex hospitality information collected by advanced technology possesses a challenge while taking the managerial decision. It demands for enhanced data reporting and analysis. Khare (2014) has mentioned that data mining allows companies utilize present reporting systems to reveal and appreciate unknown patterns in enormous record. These outlines are then assembled into data mining models and used to predict personality behavior with high precision. As an outcome of these, companies are able to assign resources and staff more effectively. Data mining techniques may help to improve students' performance in diverse ways. To achieve uppermost stage of eminence in hospitality organization is by discovering facts for prediction related to guests in a service, recognition of irregular values, forecasting of service performance etc.

According to Brodeur, M. (1998), customer satisfaction equals to division of perception of performance by expectation. The customer satisfaction survey involves two sets of questions with multiple options for estimation of perception and expectation. The value of satisfaction is derived for further actions. Though there are many scales and tools for judging the customer satisfaction, mapping the customer mind is highly mysterious. However, it is essential to convert the customer expectations in to quantifiable terms so that suitable action plan can be drawn for appropriate solutions. Brown (1998) proposed that user satisfaction being the core element of quality and the reason of crafting the interactive system for meeting the customer needs. This requires integration of hardware, software and intended users. The customer needs are entered in the metrics and people behavior is measured by system. The yield of the system can be measured as usefulness, creation, and satisfaction of the customers.

As per Infosys Viewpoint (2018), hospitality industry has introduced the element of personal service. In order to cater the ever competitive industry and stand out in the crowd, it is very necessary to find out customer preferences so that suitable service solutions can be provided. However, the large amount of customer data cannot be processed manually. Therefore hotels have incorporated AI into the customer service portfolio and overall operations. AI helps in identifying the guest preferences and seasoned decision makers can design appropriate services. AI is also instrumental in finding out expected operational risks by examining the patterns with previous data. It utilizes cognitive learning and helps in reducing human errors and potential bias in understanding situations.

According to joint research of Google \& Ellen MacArthur (2019), the AI alone can benefit the industry by US $\$ 1.8$ trillion by year 2030. The AI control on food waste alone would save a net of US \$ 
127 Billion each year. It also suggests that the holistic and overall transformation of the industry and adaption of AI would be a relatively slower but improvement in efficiency and profits margins would leverage the changes. The AI itself would refine to a robust and full proof whereby large amount of data can be converted into actionable insights.

\section{Methods}

The study is carried out to explore the various functions of travel and hospitality industry that are embedded with artificial intelligence. The study uses mixed research method and in depth study of empirical data is done to discover most significant uses of AI in hospitality and tourism sector. It is supported by first hand information received through personal interaction and inputs from industry professionals. A purposive sampling method is used for the study and a short and crisp questionnaire survey of 115 professional from hotel and tour management companies is carried out. The recorded information is sifted for analyzing logical use of AI in operations and its overall effect on most common functions. The analysis is presented in charts.

\section{Results}

Out of many facets and functions embedded with AI, the most common functions derived from extensive literature review and available resources. The responses from 115 respondents were recorded on a pretested questionnaire on three point likert scale. The questions about usage and effectiveness of AI in multiple functions are marked against suitable options. The collected data is analyzed using MS Excel and presented in tables and charts.

Table 1. Functions embedded with AI

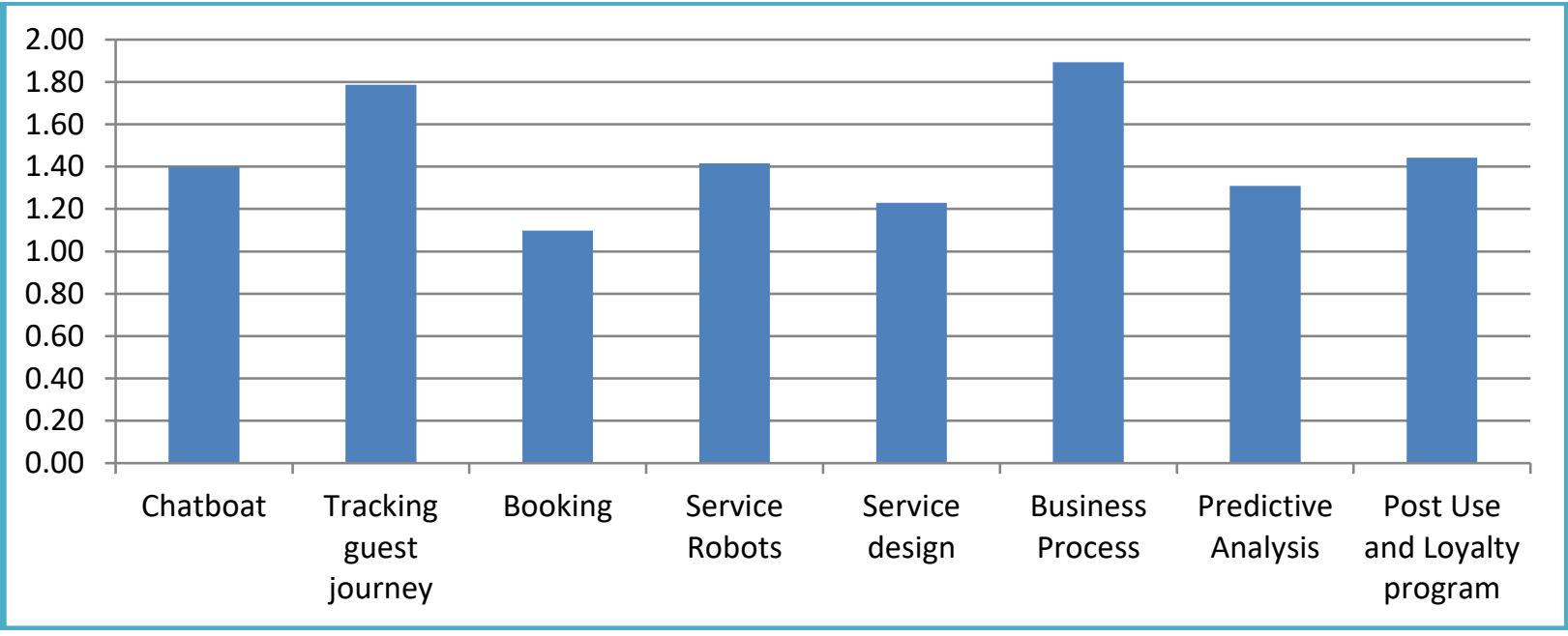

The responses collected against embedding the AI in various functions in Yes/No format.

The mean value of data against each parameter is plotted on graph. It shows the mean value above 1 , an inference can be drawn that maximum respondents have affirmative response towards implementation of AI across various functions. 
Table 2. Effectives of $\mathrm{AI}$ in operations

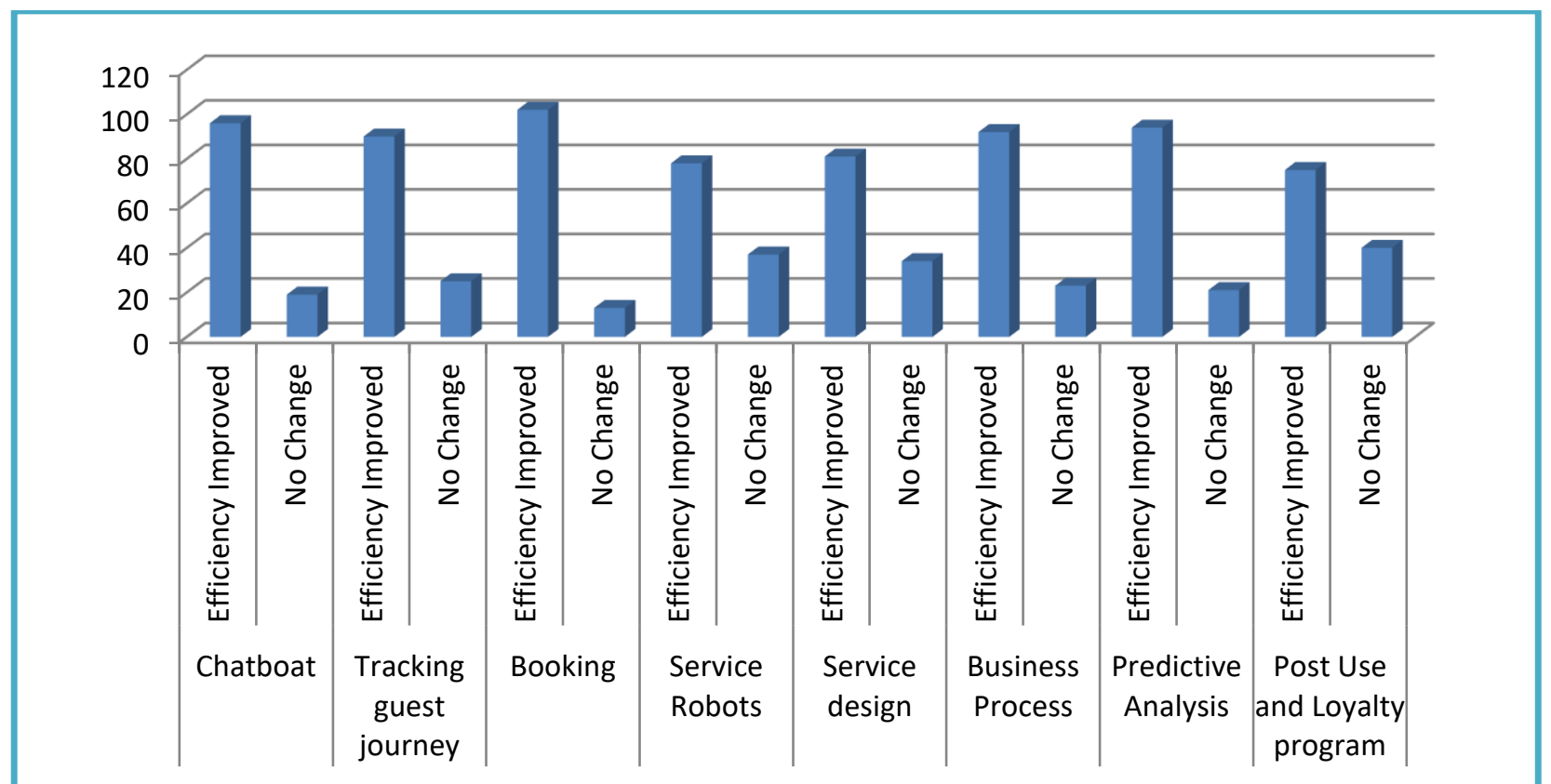

The responses collected against effectiveness of AI across various function and number of response values are plotted on graph. An inference can be drawn from above that the AI has significantly influenced the efficiency of functions to great extend. However, Service robots and Post use Loyalty Program functions has relatively less impact of AI.

\section{Discussion}

The research is related to introduce the changes in the traditional system by using AI to increase profitability, decrease unnecessary expenses and excess labor. The basic motive of embedding AI into operations is to find viable changes in the ongoing process of Hospitality Company due to which the company saves excess expenses and reduces the cost for manufacturing as well as service cost. In today's competitive world the price competition cause low profit in service industry and cost reduction is one of the best solutions to increase profitability. Now a days, the about mention concepts are highly appreciable by industry called Three R's i.e. Reduce, Reuse and Recycle. In this research the analysis of AI implementation in hospitality and tourism with feasibility. It is utmost important for companies to create a service differentiator for attracting and retaining the customer. Keeping in the mind the customer preferences and providing personalized services; use of AI simplifies the overall process. Understanding the guest preferences through complex data sets and monetizing it is made possible by appropriate AI tools.
The quantitative research has validated that the most of the hotel operations have been embedded with AI technology and likely to be embedded in all possible functions. It has also iterated that AI has significantly improved the functions and efficiency has been improved across the functions. That further supports the increasing role of $\mathrm{AI}$ in hotel and tourism operations.

Most significant and common function AI can enable is explored as below:

a) Chatboat: The most common and classic tools used widely for first point of contact with the travelers all over the world, It has a set of Frequently Asked Questions with appropriate answers fed into the system. It is programmed to answer the most common, most expected queries and questions from customers. It is an advanced form of Machine Conversation System. It also systematically analyses the intention behind each question and pre-empt the further conversation. Now days, nearly $50 \%$ of overall communication is handled by Chatboat. According to Li et al (2019), the customers are also accustomed to using chatboat and get their enquiries answered accurately. The most significant aspect of chatboat is that the matter of the discussion is more focused and provides the consumer with a more efficient experience.

b) Track the Guest Journey: AI assist hoteliers to record the guest journey from first 
instance of contact to the last one. The aim is to record, track and analyze the guest journey for revealing out the weak and augmentation points of the business. It is critical for improving customer experience. It makes AI essential to procedure and automating the data sifting through gigantic volume of data gathered.

c) Improved Booking Experience: The simple and friendlier booking process is vital advantage of AI. The AI solutions provide helps in providing the consumers with a fast-paced delivery of services, round the clock availability, booking service lots of custom made options available, and that's with less individual interference.

d) Service robots: Simpler tasks of delivering breakfast, carrying newspaper, carrying luggage, basic cleaning and some $\mathrm{f} \& \mathrm{~b}$ service in restaurant is being done with the help of robots. Germany developed the first hotel robot name Mortimer. These robots are feed with all necessary information like layout, service points, holding equipments, delivery of equipments etc and programmed for doing specific tasks.

e) Personalized service experience: Application of AI would ease out the analysis of large and complex guest data and provide insights for offering personalized service solutions. It will improve hotel services and customer satisfaction to highest level. This will also save on unnecessary and unidirectional planning of services, thus as a result will optimize the performance of hotel operations.

f) Business processes optimization: According to latest study by Oracle, AI has been already in use in various travel \& hospitality functions that includes housekeeping, front office, f\&b service, reservations, customer history, cost reporting and so on. It is estimated that it has over $89 \%$ of hoteliers have registered savings in hotel operations. AI has leverage advance control, accuracy, effective monitoring, data management and reduced human resource.

g) Predictive Analysis: Google \& Ellen MacArthur (2019) study suggests that artificial intelligence based systems will be able to read huge data and analyze the patterns and habits associated with certain groups of people. AI deals with human character of intelligence which performs learning and logical reasoning and is capable of bringing in fourth industrial revolution. It will enhance human decision making with predicative analysis. It includes the expected occupancy, room revenue, $f \& b$ revenue as well as expenses. The guest related prediction will help in improving service quality especially considering the tech savvy millennial customer.

h) Improved bottom line: As per ehotelier.com report (2020) earlier studies have listed various benefits of AI embedded operations and it improved efficiency. It gives innovative capabilities and improves guest satisfaction. On other hand, it helps in reducing the operating costs of hotel operations through accuracy, reduced wastage and thefts, lesser touch points and predictions about customer requirements. IT enables two dimensional communications through automated systems and improved feedback system making it guest centric enterprise.

i) Post voyage \& loyalty program: AI has been effectively used for tracking the guest feedback across various platforms after the guest leaves the premises. It also enables tracking the guest patronage and an advanced loyalty program can be managed with the help of insights from AI.

However, like other technologies, AI also has to face some limitations. It may be due to definite reasons as listed below:

a. Apathy towards adaption of AI in operations.

b. Poor implementation and training to staff on effective utilization of AI.

c. Improper and inadequate adaption of AI in hotel operations.

d. Limited access to guest and revenue data.

e. Limited or insufficient knowledge of AI benefits and poor understanding.

f. Selection of wrong or incapable IT vendor.

These limitations may act like challenges but considering the AI solutions and muscle it can leverage to tourism and hotel management, it is utmost important to overcome these obstacles and use AI to witness dramatic transformation of operations. This study also opens up the avenues for further studies in different markets and varied operations of hospitality and tourism industry.

6. References

1) Brodeur M.(1998), Satisfaction, dissatisfaction and complaining behavior with consumer services and intangible 
products. Indiana University Graduate School of Business, DBA dissertation.

2) Brown, M G, (1991), Baldrige Award Winning Quality: How to Interpret the Malcom Baldrige

3) Award Criteria, Milwaukee, WI: ASQC Quality Press

4) IDC FutureScape: Worldwide IT Industry 2019 Predictions

5) Infosys Viewpoint (2018), published by Infosys Inc

6) Khare Nidhi (2014), A Study of Applications of Data Mining Techniques in Higher Education, Confluence proceedings

7) Koranne Sameer (2020), A methodical assessment of Servqual model, Palarch's Journal Of Archaeology of Egypt/Egyptology 17(12), ISSN 1567-214x

8) Li, Bai, Jieng $\mathrm{N}$, Sham J, Shi H, Fazal H, (2019), Real-World Conversational AI for Hotel Bookings, IEEE Second International Conference on Artificial Intelligence for Industries (AI4I)

9) International Conference on Artificial Intelligence for Industries (AI4I).

10) Minsky Marvin, (1961) Steps toward Artificial Intelligence, Proceedings of IRE, Vol 49 Issue 1

11) SITA Air Transport Insights report (2017)

12) www.ellenmacarthurfoundation.org/publicat ions/artificial-intelligence-and-the-circulareconomywww.insights.ehotelier.com/insight s/2020/05/11/artificial-intelligence-improvecustomer- www.oracle.com, Oracle report (2019) How AI enhances hotel guest experience experience-in-your-hospitalitybusiness/ 